\title{
Anesthesia for awake craniotomy: A retrospective study
}

\author{
Prabhat Kumar Sinha, Thomas Koshy, P. Gayatri, V. Smitha, Mathew Abraham*, \\ Ramesh Chandra Rathod \\ Departments of Anesthesiology and *Neurosurgery, Sree Chitra Tirunal Institute for Medical Sciences and Technology, Trivandrum, Kerala, \\ India
}

Context: Awake craniotomy is increasingly performed the world over. We share our experience of performing craniotomy awake with our anesthetic protocol. Aims: To evaluate and analyze the anesthesia records of the patients who underwent awake craniotomy at our institution. Settings and Design: University teaching hospital, Retrospective study. Materials and Methods: We reviewed records of the 42 consecutive patients who underwent awake craniotomy under conscious sedation using Fentanyl and Propofol infusion until December 2005. The drugs were titrated (Bispectral monitoring was used in 16 patients) to facilitate intermittent intraoperative neurological testing. All patients received scalp blocks with a mixture of bupivacaine and lignocaine with adrenaline. Haloperidol and ondansetron were administered in all patients at induction of anesthesia. Results: All patients completed the procedure. One patient each needed endotracheal intubation and LMA for airway control during closure, while another required CPAP perioperatively because of desaturation to $<80 \%$. There was significantly decreased use of anesthetics $(P<0.001)$ and a trend towards reduction in complications (e.g. respiratory depression and deep sedation) ( $P>0.05$ ) with the use of BIS as compared to without BIS. Intraoperative complications were hypertension (19\%), tight brain (14.2\%), focal seizure $(9.5 \%)$ respiratory depression $(7.1 \%)$, deep sedation $(7.1 \%)$, tachycardia $(7.1 \%)$ and bradycardia. Two patients desaturated to $<95 \%$. $23.8 \%$ patients developed transient neurological deficits. The most frequent postoperative complications were PONV (19\%) and seizures (16.6\%). Conclusions: With the use of advanced monitoring and newer anesthetics, awake craniotomy is a relatively safe procedure with an accepted rate of complications.

Key words: Anesthesia, awake craniotomy, bispectral index, complication, fentanyl, haloperidol, monitoring, propofol, regional anesthesia, scalp block

There is an increasing trend towards performing craniotomy awake. This is done either for resection of the tumor located in the eloquent areas of the brain or for localization and resection of seizure focus. The challenge before the anesthesiologist is to provide adequate analgesia and sedation, hemodynamic and respiratory stability and a safe airway, with awake, cooperative patients for neurological testing.

In our institution, we have been performing awake craniotomy for the last five years. The aim of the study is to analyze our data of anesthetic management of the patients in whom awake craniotomy was performed at our institute until December 2005.

\section{Materials and Methods}

We retrospectively reviewed the anesthetic management and incidence of complications of all patients who had undergone awake craniotomy (excluding patients undergoing awake stereotactic procedures or open brain biopsies). The study was approved by our institute for a confidential review of the patient notes, which were to be taken from medical record department. The anesthetic records, operation notes and the postoperative notes were examined and the incidence of complications [deep sedation (defined as drug-induced depression of consciousness during which patients cannot be easily aroused but respond purposefully following repeated or painful stimulation), hypertension (systolic blood pressure $>30 \%$ of base value), tachycardia and bradycardia (heart rate $>140$ and $<50$ bpm respectively), respiratory depression ( $\mathrm{RR}<8$ breath/min), desaturation $\left(\mathrm{SpO}_{2}<95 \%\right)$ and nausea and vomiting (PONV)] was recorded. Any patient developing neurological deficits (transient or permanent) was also noted. Transient neurological deficits (consist mainly of sensory or motor deficit) are defined as the neurological deficit, which is

Presentation at a meeting:

$51^{\text {st }}$ Annual Conference of the Indian Society of Anesthesiologists, Bhubaneshwar, Orissa, India, December 2003 
either improved or recovered fully during the first six weeks of follow-up. All other neurological deficits are classified as permanent neurological deficits. Patients were selected for the surgery on the basis of their requirement for awake craniotomy as well as patient's affirmation to cooperate fully with the procedure. The precise indications for surgery were (i) lesions near the eloquent cortex determined by MRI, (ii) patients with overlap of functional areas with the lesion based on fMRI, (iii) clinical evidence of functional overlap with lesions meant for resection, (iv) conditions where an intentional but acceptable deficit is planned and (v) when an extra-operative stimulation (based on a subdural grid) showed functional areas in or in close proximity with epileptogenic zones.

Anesthesiologists involved with the procedure visited these patients one day prior to surgery. The main purpose of the preoperative visit included taking patients into full confidence and a lengthy explanation of the chronological sequence of the procedure and the points during which pain or discomfort might be experienced. Patients were kept fasted since midnight and were administered clonidine $(4 \mu \mathrm{g} / \mathrm{kg})$, ranitidine, atenolol (25 mg) and double the dose of anticonvulsants orally in the morning, however, anticholinergics were avoided. Any cardiac medications were continued as usual.

Preparation in the operating room involved making the patient as comfortable as possible. Operating room temperature was kept warm or alternatively, warming blanket/forced air warming device was used. $\mathrm{O}_{2}$ was started via nasal cannula (2-4 LPM), once patients arrived in the operating room, an intravenous cannula, an arterial cannula in one of the radial artery and a central venous catheter into the patient's antecubital vein were sited under local anesthesia. Patient was further monitored with ECG, end tidal carbon dioxide $\left(\mathrm{EtCO}_{2}\right)$ pulse oximetry $\left(\mathrm{SpO}_{2}\right)$, urine output and Bispectral index (BIS) (as per the availability). All patients were monitored with electrocorticography (ECoG), somatosensory evoked potential and direct cortical stimulation, however, motor evoked potential was not used.

Scalp nerve blocks were performed bilaterally in all patients while awake by a mixture of $0.25 \%$ bupivacaine and 1\% lignocaine with 1: 200000 adrenaline $(2-5 \mathrm{ml}$ at each infiltration site). The nerves infiltrated were supratrochlear and supraorbital, auriculotemporal, greater and lesser occipital and great auricular. All patients received fentanyl (approximately 25-50 $\mu \mathrm{g}$ ) i.v. before scalp blocks. Patient's head was positioned either on horse-shoe head holder or on pins as per the requirement. The same mixture was used at pin insertion site (1.5 $\mathrm{ml}$ at each site) and at skin incision site. Total dose of local anesthetics was carefully calculated to avoid using above maximum permissible limit. Patients were induced with intravenous fentanyl (0.5-1.0 $\mu \mathrm{g} / \mathrm{kg}$ ), propofol (1-2 mg/kg) and midazolam (1$2 \mathrm{mg}$ ) followed by infusion of fentanyl $(0.5-2 \mu \mathrm{g} / \mathrm{kg} / \mathrm{hr})$ and propofol (1-5 mg/kg/hr). All patients also received intravenous haloperidol (2.5-5 $\mathrm{mg}$ ) at induction. The drugs were so titrated to keep BIS value above 60 (if used) and to facilitate intraoperative neurological testing without compromising patient's airway and respiration. Also, ondansetron (4 $\mathrm{mg}$ ) was administered before start of surgery and at closure of the duramater. To treat tachycardia and/or hypertension, metoprolol was administered and anesthesia was deepened. Furthermore, intravenous diclofenac (50-75 mg)/ tramadol (50-100 mg) were administered as per patient's response to pain. Again local anesthetic was infiltrated at skin incision site after closure of the dura. All patients received antibiotic, corticosteroids, anti-epileptic drugs and mannitol during the intraoperative period. Some patients required furosemide. Postoperative pain was controlled with intramuscular diclofenac and/or intravenous tramadol. We used 'conscious sedation' technique (in which use of drug(s) produce(s) a state of depression of the CNS enabling treatment to be carried out, but during which verbal contact with the patient is maintained throughout the period of sedation ${ }^{[1]}$ ) in all of our patients.

Data were analyzed (SPSS version 11.0) using student's t-test and Fisher's exact test wherever appropriate. A P value of $<0.05$ was considered as significant.

\section{Results}

Forty-two patients had undergone awake craniotomy. The pathological diagnosis, demographic and operative characteristics as well as complications are shown in Tables 1, 2, 3 and 4. Of 42 patients, 11 were operated for refractory epilepsy while the rest for tumor resection. Two patients required airway intervention. One patient needed continuous positive airway pressure (CPAP) during surgery and up to $2 \mathrm{~h}$ postoperatively. The most frequent intraoperative complications observed were hypertension and tight brain.

Bispectral index was used in 16 patients. Use of propofol and fentanyl were significantly reduced where BIS was used as compared to no BIS group $(P<0.001)$ [Table 5]. No patients in the BIS group had either respiratory depression or deep sedation. There was a lack of statistical significance between the two groups as regards the complications observed in this study (e.g. respiratory depression, deep sedation and occurrence of intra- and/or postoperative seizure) $(\mathrm{P}>0.05)$ [Table 5].

\section{Discussion}

Awake craniotomy allows cortical mapping with patient cooperation and helps prevent neurological dysfunction. 
Table 1: Pathological diagnosis

\begin{tabular}{lc}
\hline Pathological diagnosis & $\begin{array}{c}\text { No. of patients } \\
\text { (n=42) }\end{array}$ \\
Left frontal astrocytic glioma & 8 \\
Left fronto-parietal low-grade glioma & 2 \\
Left temporal glioma & 2 \\
Left parieto-occipital glioma & 2 \\
Left parietal venous angioma & 1 \\
Left medial frontal fibrillary astrocytoma Grade II & 1 \\
Left posterior frontal astrocytoma & 2 \\
Left insular glioma & 3 \\
Left inferior frontal pre-motor astrocytoma & 1 \\
Grade II & \\
Left pre-motor cavernomatous malformation & 1 \\
Right pre-motor (posterior frontal region) & 2 \\
mixed glioma & 1 \\
Right fronto-parietal glioma & 2 \\
Right parietal low-grade glioma & 2 \\
Right frontal astrocytoma Grade II & 1 \\
Right parietal DNET with ganglioglioma & \\
component & 3 \\
MR CPS with left frontal cortical dysplasia & 2 \\
MR CPS with right frontal cortical dysplasia & 1 \\
MR CPS with left perisylvian gliotic scar & 1 \\
MR CPS of right hemispheric origin & 1 \\
MR extra-temporal CPS of left frontal origin & 1 \\
(gliosis) & 2 \\
MR CPS of left fronto-insular cortical dysplasia & \\
Rasmussen's encephalitis with medically & \\
refractory CPS & \\
\hline DNET Dysembroplasic neuroeptheiat tumor CPS: Comptex patta sezure;
\end{tabular}

DNET: Dysembroplastic neuroepithelial tumor; CPS: Complex partial seizure; MR: Medically refractory

Table 2: Demographic and operative characteristics
Sex; M:F

Age; years

Weight; kg

Surgery time; $\min$

Anesthesia time; min

Total dose of fentanyl used; $\mu \mathrm{g}$

Total dose of propofol used; $\mathrm{mg}$

Use of BIS; $n$

Total dose of $0.25 \%$ bupivacaine used; $\mathrm{ml}$

$\mathrm{O}_{2}$ used; lit/min

Time since admission to discharge; days

Values are interquartile range [range], mean (SD) or number

Table 3: Intraoperative complications during awake craniotomy Values are number of patients (\%), $n=42$

\begin{tabular}{lc}
\hline Hypertension & $8(19.0)$ \\
Tight brain & $6(14.2)$ \\
Focal seizure & $4(9.5)$ \\
Increase in EtCO2 $(>50 \mathrm{~mm} \mathrm{Hg})$ & $4(9.5)$ \\
Respiratory depression $(\mathrm{RR}<8$ breath/min) & $3(7.1)$ \\
Tachycardia $(\mathrm{HR}>140$ beats/min) & $3(7.1)$ \\
Bradycardia $(\mathrm{HR}<50$ beats/min) & $2(4.8)$ \\
Deep sedation & $3(7.1)$ \\
Desaturation $(\mathrm{SpO} 2<95 \%)$ & $2(4.8)$ \\
PONV & 0
\end{tabular}

$\overline{\mathrm{RR}}=$ Respiratory rate, $\mathrm{HR}=$ Heart rate, $\mathrm{PONV}=$ Postoperative nausea and vomiting
Table 4: Postoperative complications following awake craniotomy Values are number of patients $(\%)(n=42)$

Neurological deficit

Transient

Permanent

$10(23.8)$

Nausea and/or vomiting

Seizure

$8(19.0)$

$7(16.6)$

$2(4.8)$

Digital ischemia
Pneumocephalus

Despite widespread use of this technique, its optimal anesthetic management remains a challenge. ${ }^{[2]}$ It requires (i)sufficient depth of anesthesia, (ii)full cooperation and consciousness during cortical mapping, (iii)smooth transition between anesthesia and consciousness, (iv)adequate airway and (v)patient immobility. ${ }^{[2]}$ To satisfy these requirements, several techniques have been described $^{[3-5]}$ that included sedation and local anesthesia under monitored anesthesia care (MAC) also called conscious sedation, asleep-awake-asleep technique using laryngeal mask airway (LMA) or endotracheal intubation and breathing spontaneously, and total intravenous anesthesia with controlled ventilation using endotracheal intubation during opening and closure of the skull and performing extubation during neurological assessment. We used conscious sedation technique in all patients.

Currently, a number of agents are available that provide adequate analgesia/sedation, minimally interfere with ECoG and allow for rapid recovery when conscious is required. Propofol infusion is one of them ${ }^{[6]}$ or in combination with short-acting narcotic. ${ }^{[7]}$ Propofol offers the advantages of being a short-acting sedative with antiemetic and amnestic properties in sedative doses, ${ }^{[8-9]}$ as well as its ability to reduce the incidence of intraoperative seizures without compromising the quality of ECoG monitoring during awake craniotomy for seizures. ${ }^{[10]}$ Furthermore, stopping the propofol 5 min before ECoG did not affect the monitoring. ${ }^{[10]}$ Propofol sedation in combination with fentanyl was compared to the more traditional fentanyl-droperidol regimen in patients operated for seizures by Herrick et al. and it was found that while levels of intraoperative sedation, patient satisfaction and cognitive functions were similar in both groups, the incidence of transient hypoventilation was more among the propofol-treated patients. ${ }^{[11]}$ Propofol can also be administered through target control infusions. ${ }^{[12]}$

Gignac et al. ${ }^{[13]}$ compared fentanyl, sufentanil and alfentanil, in addition to droperidol during awake craniotomy for epilepsy. Sufentanil and alfentanil offer some theoretical advantages over fentanyl like the conditions for the surgery, ECoG monitoring and for stimulation testing. ${ }^{[13]}$ The main complications 
Table 5: Comparison of data using bispectral index and without bispectral index. Values are mean (SD) or number (\%) ( $\mathrm{n}=42)$

Total dose of fentanyl used; $\mu \mathrm{g}$ Total dose of propofol used; mg Incidence of respiratory depression (\%) Incidence of deep sedation (\%) Incidence of Seizure

Intraoperative

Postoperative

Total

$\mathrm{BIS}=$ Bispectral Index

$\begin{array}{ccc}\begin{array}{c}\text { With BIS } \\ (\mathbf{n = 1 6 )}\end{array} & \boldsymbol{P} \text { value } & \text { Confidence Interval } \\ 129.6 \pm 56.3 & <0.000 & 103.1,173.1 \\ 185.6 \pm 90.2 & <0.000 & 141.0,283.9 \\ 0 & 0.27 & \\ 0 & 0.27 & \\ & & \\ 1 & 1.00 & \\ 2 & 0.69 & \\ 3 & 0.48 & \end{array}$

Without BIS

$(\mathrm{n}=\mathbf{2 6})$

$67.7 \pm 49.6$

$398.1 \pm 138.7$

$3(10.7)$

$3(10.7)$

3

5

8
0.69 found in their study were PONV, seizures, desaturation, increase in $\mathrm{EtCO}_{2} \cdot{ }^{[13]}$ Despite rise in $\mathrm{EtCO}_{2}$, none of the their patients had "tight" brain upon dural opening. ${ }^{[13]}$ In contrast, $14.2 \%$ of our patients had "tight brain". The higher incidence of this could be related to poorly defined "tight brain" and subjective variation in assessment of its occurrence. Remifentanil has also been used satisfactorily by Johnson et al. ${ }^{[14]}$ and a combination of propofol-remifentanil infusion has gained popularity in the management of awake craniotomies. ${ }^{[15]}$

We performed awake craniotomy with fewer complications than reported by others. Respiratory depression and deep sedation were attributed to the use of increased dose of anesthetics. However, none of the 16 patients in whom BIS was used had respiratory depression or deep sedation. Furthermore, incidences of PONV, desaturation and increase in $\mathrm{EtCO}_{2}$ were lower in our series than Gignac et al.'s study, ${ }^{[13]}$ however, incidence of seizure was higher in our study. This can be partly attributed to the learning curve for the team involved as confirmed by our data which showed fewer patients had postoperative seizure during surgery of next 21 patients as compared to first 21 patients ( 2 vs. 5) $(P=0.09)$, however, more patients had intraoperative seizure who were operated during the later half (1 vs. 3) $(P=0.42)$. Recently, Skucas et al. reported higher incidence of intraoperative complications in sleepawake-asleep technique than in those where general anesthesia was administered. ${ }^{[16]}$ Our incidence of complications is comparable to that of Skucas et al.'s study except for the incidence of seizure which was higher in our study. Furthermore, surgical and anesthetic procedures in our series also took a relatively longer time than expected, ${ }^{[3]}$ which can again be attributed to the initial learning curve. Time to complete the procedure, however, decreased progressively. Two patients had bradycardia intraoperatively which is occasionally seen during epilepsy surgery. ${ }^{[17]}$

Hospital stay for awake craniotomy was $13.3 \pm 4.2$ (mean $\pm S D$ ) in our series. It is difficult to prove whether our protocol facilitated shorter hospital stay as there were no comparable data and was beyond the aim of this study. However, Blanshard et al have concluded that patients can be discharged on the same or next day after surgery provided stringent criteria for patient selection is made. ${ }^{[18]}$

We avoided anticholinergic as a premedication as it causes unpleasant dryness of mouth while patient remains awake. However, we routinely administer clonidine, ranitidine and antiemetics before scheduled surgery. Our patients had stable hemodynamic on arrival to operation room which is expected in view of clonidine's ability to induce mild sedation, hemodynamic stability and an analgesic and antiemetic effect. ${ }^{[19]}$

Haloperidol used in this series is a butyrophenone similar to droperidol with longer duration of action that lacks significant alpha-adrenergic antagonistic effects with no reported cardiac toxicity. Its clinical effect is characterized by trance-like (cataleptic) immobility in an outwardly tranquil patient who is dissociated and indifferent to the external surroundings. It has an antiemetic effect at considerably lower doses that are generally used for the treatment of psychosis or the control of agitation. ${ }^{[20]}$ The only concern with haloperidol is its potential to cause neurological adverse effects at low, antiemetic doses and in rare instances, can cause extrapyramidal symptoms. ${ }^{[20]}$ Fortunately, none of our patients had these because of its use. It may also cause sedation, especially if used in large doses. To the best of our knowledge, ours is the first reports of its use in a considerable number of patients subjected to awake craniotomy.

We administered double the dose of antiepileptic drugs in the morning of the surgery and another dose once ECoG recording was over. Despite this protocol, we encountered intraoperative focal seizures and postoperative generalized seizure in $9.5 \%$ and $16.6 \%$ of cases respectively. Use of double the dose of antiepileptic drugs preoperatively did not interfere in ECoG recording in any patient and can be used especially in those patients who are at greater risk of developing it. Once seizures occur they can be controlled by administering another dose of antiepileptic drugs and/or intravenous midazolam. However, midazolam may interfere with neurological testing. Keifer et al. advocated application of ice slush irrigation at the surgical field to treat seizures. ${ }^{[21]}$ Several investigators 
demonstrated predominant anti-convulsant activity associated with propofol, ${ }^{[22-23]}$ however, we could not say with certainty whether its use indeed helped in decreasing the incidence of seizure. Effects different newer opioids (viz. fentanyl, sufentanil and alfentanil) on the ECoG and central nervous system are similar. ${ }^{[24]}$ However, the differences in the clinical effects of these opioids are due to their different pharmacokinetic properties. ${ }^{[25-26]}$ Tempelhoff et al. described increased fentanyl requirement for those who are on chronic antiepileptic drugs during anesthesia for craniotomy, ${ }^{[27]}$ however, we did not observe increased requirement in our patients. This could possibly be because of (i)most of the patients were not on chronic antiepileptic drugs, (ii)use of diclofenac and/or tramadol, (iii)use of clonidine ${ }^{[19]}$ and (iv)use of BIS.

Bispectral index (Aspect Medical Systems, Natick, MA) was used to measure depth of anesthesia. It has been shown to correlate well with the hypnotic component of anesthesia and can be used as a guide to the administration of volatile and intravenous anesthetics. ${ }^{[28]}$ It can also vary in response to noxious stimuli such as the application of a head holder. ${ }^{[29]}$ This is the first large series of using BIS in awake craniotomy. We found that with the use of BIS, dose of propofol and fentanyl were significantly reduced as compared to no BIS monitored patients [Table 5]. This finding is consistent with many other researchers where it is documented that BIS significantly reduces consumption of many anesthetic agents, ${ }^{[30]}$ however, overall cost of anesthesia might be increased because of high cost of BIS electrode. ${ }^{[31]}$ We advocate a further study to analyze the 'cost-effectiveness factor' by using BIS in this subset of patients. Furthermore, none of our patients who were monitored with BIS had respiratory depression or deep sedation, which entails its usefulness in the present context. Hans et al. described the use of BIS in a patient for awake craniotomy using target-controlled infusion of propofol and remifentanil. ${ }^{[32]}$

Respiratory compromise is possible when using a technique with an unsecured airway. Transition from conscious sedation to deep sedation is likely if dose of the anesthetics are not titrated precisely or it occurs even with the use of shorter-acting opioids. ${ }^{[21]}$ Emergency control of airway is of paramount importance in these situations. One should choose a technique where controlled ventilation can be achieved easily without disturbing the surgical field. This may sometimes be difficult when the head of the patient is inaccessible or fixed on pins. Airway should be controlled to earliest possible opportunity to avoid ventilatory depression and hypercarbia and the consequences thereof on cerebral hemodynamics. ${ }^{[33]}$ One should also remember that the patients need to be awake and possibly be extubated for neurological testing and hence the anesthetics chosen for emergency airway control should have shorter duration of action and should be such as can easily be reversed. Another major problem encountered in spontaneously breathing but sedated patients is that $\mathrm{EtCO}_{2}$ cannot be completely controlled and this is the reason why four of our patients $\left(9.5 \%\right.$ ) had their $\mathrm{EtCO}_{2}>50 \mathrm{mmHg}$. This could be because of deepening the anesthesia to obtund the intense surgical stimulation. ${ }^{[13]}$

One of our patients required endotracheal intubation as this patient became restless and developed bodily pain for lying supine for a prolonged period. Fortunately, the airway was secured with intubating LMA (ILMA) using succinylcholine and thiopental sodium i.v. without much difficulty as the patient's head was on horseshoe head holder. Another patient required LMA during dura closure as this patient became apnoeic. No difficulty was encountered while securing the airway (using propofol without muscle relaxant) despite the head being on pins. If head is fixed on pins, intubation can be performed using fibreoptic (FOB) guided endotracheal intubation or classical LMA/ILMA/cuffed oropharyngeal airway insertion or by CPAP. ${ }^{[2,16,33-34]}$ One patient required CPAP perioperatively because of desaturation during surgery. Continuous positive airway pressure was delivered through nasal trumpet during intraoperative period and through face-mask in the postoperative period. Skucas et al., ${ }^{[16]}$ also reported airway/ventilation complications in $1.8 \%$ of their cases as compared to $7.1 \%$ in this series. Yamamoto et al. have also used noninvasive ventilation in two patients scheduled for awake craniotomy and advocated its use in certain circumstances. ${ }^{[2]}$

Prevention of PONV is of paramount importance while the patient is in conscious sedation state. Fortunately, none of our patients either nauseated or vomited intraoperatively. However, $19 \%$ of patients had incidence of PONV. Other authors reported incidence of PONV as high as $50 \% .{ }^{[13]}$ The difference in this series could be attributed to adequate pain relief (not analyzed in the present series), use of antiemetic, clonidine, haloperidol and propofol perioperatively.

Despite continuous neurological monitoring, 23.8\% developed transient neurological deficits in our series with no reported incidence of permanent neurological deficit. Taylor et al. reported $8.5 \%$ and $4.5 \%$ incidence of transient and permanent neurological deficits respectively. ${ }^{[3]}$ Higher incidence of transient neurological deficit in our series could be because of our different approach of performing awake craniotomy in which surgical resection was maximized at the calculated risk of development of mild or acceptable neurological deficits, learning curve and fewer patients recruited as compared to Taylor et al., (42 vs. 200). ${ }^{[3]}$

The youngest patient operated in our series was a 12year-old boy. This patient was properly evaluated by the psychologist. He was found to be very cooperative and showed high level of endurance and was able to concentrate and perform specific frustrating tasks for 
longer duration and inference was drawn that the child can undergo the procedure safely. This entails that the procedure could be safely conducted even in younger children. ${ }^{[35]}$

Using this protocol, awake craniotomy is performed safely. Furthermore, our protocol has been improved upon by using BIS, avoiding anticholinergic as a premedicant, using $\beta$-blockers intraoperatively to treat tachycardia and/or hypertension and use of antiemetics.

In summary, judicious use of anesthetics and its adjunct allows satisfactory conditions for awake craniotomy without development of significant complications in small series of this retrospective study.

\section{Acknowledgment}

We gratefully acknowledge the support provided by the Director, SCTIMST for his kind permission for the publication of the manuscript.

\section{References}

1. Skelly AM. A nalgesia and sedation. In: Watkinson A, Adam A, editors. Interventional Radiology. Radcliffe M edical Press: Oxford; 1996. p. 3-11.

2. Yamamoto $\mathrm{F}, \mathrm{K}$ ato $\mathrm{R}$, Sato J, N ishino T. Anesthesia for awake craniotomy with non-invasive positive pressure ventilation. $\mathrm{Br} J$ Anaesth 2003;90:382-5.

3. Taylor MD, Bernstein M. Awake craniotomy with brain mapping as the routine surgical approach to treating patients with supratentorial intraaxial tumors: A prospective trial of 200 cases. I Neurosurg 1999;90:35-41.

4. Sarang A, Dinsmore J. Anaesthesia for awake craniotomy - evaluation of a technique that facilitates awake neurological testing. $\mathrm{Br}$ J Anaesth 2003;90:161-5.

5. H uncke K, Van de Wiele B, Fried I, Rubinstein E H. The asleep-awakeasleep anesthetic technique for intraoperative language mapping. $\mathrm{N} \mathrm{eu}-$ rosurgery 1998;42:1312-7.

6. Silbergeld DC, Mueller WM, Colley PS, Ojemann GA, L ettich E. U se of propofol (Diprivan) for awake craniotomies: Technical note. Surg Neurol 1992;38:271-2.

7. Archer DP, McK enna J M, Morin L, Ravussin P. Conscious-sedation analgesia during craniotomy for intractable epilepsy. A review of 354 consecutive cases. Can J Anaesth 1988;35:338-44.

8. B orgeat A, Wilder-Smith OH , Saiah M, R ifat K. Subhypnotic doses of propofol possess direct antiemetic properties. Anesth Analg 1992;74:539-41.

9. Smith I, Monk TG, White PF, Ding Y. Propofol infusion during regional anesthesia: Sedative, amnestic and anxiolytic properties. Anesth Analg1994;79:313-9.

10. H errick IA, Craen RA, G elb AW, M CL achlan RS, Girvin J P, Parrent AG, eal. Propofol sedation during awake craniotomy for seizures: $E$ lectrocorticographic and epileptogenic effects. Anesth Analg 1997;84:1280-4.

11. Herrick IA, Craen RA, Gelb AW, Miller LA, Kubu CS, Girvin J P, \& al. Propofol sedation during awake craniotomy for seizures: Patient controlled administration versus neurolept analgesia. Anesth Analg 1997;84:1285-91.

12. Danks RA, Rogers M, Aglio LS, Gugino LD, B lack PM. Patient tolerance of craniotomy performed with patient under local anesthesia and monitored conscious sedation. N eurosurgery 1998;42:28-36.

13. Gignac $E$, Manninen PH, Gelb AW. Comparison of fentanyl, sufentanil and alfentanil during awake craniotomy for epilepsy. Can J Anaesth 1993:40:421-4.

14. J ohnson K B , E gan TD. Remifentanil and propofol combination for awake craniotomy: Case report with pharmacokinetic stimulation. J N eurosurg
Anesthesiol 1998;10:25-9.

15. B erkenstadt $H$, Perel A, H adani M, U nofrievich I, Ram Z. Monitored anesthesia care using remifentanil and propofol for awake craniotomy. J Neurosurg Anesthesiol 2001;13:246-9.

16. Skucas AP, Artru AA. Anesthetic complications of awake craniotomies for epilepsy surgery. Anesth A nalg 2006;102:882-7.

17. Sinha PK, N eema PK, Manikandan S, Unnikrishnan K P, Rathod $\mathrm{RC}$. B radycardia and sinus arrest during saline irrigation of the bare brain structures following epilepsy surgery. J Neurosurg Anesthesiol 2004;16:160-3.

18. B lanshard H J, Chung F, M anninen PH, Taylor MD, B ernstein M. A wake craniotomy for removal of intracranial tumour: Considerations for early discharge. Anesth Analg 2001;92:89-94.

19. Sanderson PM, Eltringham R. The role of Clonidine in anaesthesia. H osp M ed 1998;59:221-3.

20. Buttner M, Walder B, von E Im E, Tramer MR. I s low-dose haloperidol a useful antiemetic? A meta-analysis of published and unpublished randomized trials. Anesthesiology 2004;101:1454-63.

21. K eifer J C, D entchev D, L ittle K, Warner DS, Friedman AH, B orel CO. A retrospective analysis of a remifentanil/propofol general anesthetic for craniotomy before awake functional brain mapping. Anesth Analg 2005;101:502-8.

22. E brahim ZY, Schubert A, Van N ess P, Wolgamuth B, A wad I. The effect of propofol on electroencephalogram of patients with epilepsy. Anesth Analg 1994;78:275-9.

23. Samra SK, Sneyd J R, Ross DA, H enry TR. E ffects of propofol sedation on seizures and intracranially recorded epileptiform activity in patients with partial epilepsy. Anesthesiology 1995;82:843-51.

24. Scott J C, Ponganis KV, Stanski DR. EEG quantification of narcotic effect: The comparative pharmacodynamics of fentanyl and alfentanil. Anesthesiology 1985;62:234-41.

25. B ovill J G, Sebel PS, B lackburn CL, Oei-Lim V, H eykants J]. The pharmacokinetics of sufentanil in surgical patients. Anesthesiology 1984;61:502-6.

26. B ower $\mathrm{S}, \mathrm{H}$ ull CJ . Comparative pharmacokinetics of fentanyl and alfentanil. Br J Anaesth 1982;54:871-7.

27. Tempelhoff $R$, Modica PA, Spitznagel $E L / r$. Anticonvulsant therapy increases fentanyl requirements during anesthesia for craniotomy. Can J Anaesth 1990;37:327-32

28. Sebel PS, Lang E, Rampil IJ, White PF, Cork R, J opling M, \& al. A multicentre study of bispectral electro-encephalogram analysis for monitoring anesthetic effect. Anesth Analg 1997;84:891-9.

29. $H$ ans $P, B$ richant J F, D ewandre PY, B orn J D, L amy M. E ffects of two calculated plasma sufentanil concentrations on the hemodynamic and bispectral index responses to Mayfield head holder application. J N eurosurg Anesthesiol 1999;11:81-5.

30. Gurses E, Sungurtekin H, Tomatir E, Dogan H. Assessing propofol induction of anesthesia dose using bispectral index analysis. Anesth Analg 2004;98:128-31.

31. L uginbuhl M, Wuthrich S, Petersen-Felix S, Zbinden AM, Schnider TW. Different benefit of bispectal index (BIS) in desflurane and propofol anesthesia. Acta Anaesthesiol Scand 2003;47:165-73.

32. $H$ ans $P, B$ onhomme $V, B$ orn J D, M aertens de N oordhoudt A, B richant J F, D ewandre PY. Target-controlled infusion of propofol and remifentanil combined with bispectral index monitoring for awake craniotomy. Anaesthesia 2000;55:255-9.

33. Lobo FA, Amorim P. Anesthesia for craniotomy with intraoperative awakening: How to avoid respiratory depression and hypertension? Anesth Analg 2006;102:1593-4.

34. Tongier WK, J oshi GP, L anders DF, Mickey B. U se of the laryngeal mask airway during awake craniotomy for tumor resection. J Clin Anesth 2000;12:592-4.

35. K limek M, Verbrugge SJ, R oubos S, van der M ost E, Vincent AJ, K lein $\mathrm{J}$. A wake craniotomy for glioblastoma in a 9 -year-old child. A naesthesia 2004;59:607-9.

Accepted on 02-04-2007

Source of Support: Nil, Conflict of Interest: None declared. 